\title{
TWO-BODY MATRIX ELEMENTS FROM A MODIFIED SURFACE DELTA INTERACTION
}

\author{
P. W. M. GLAUDEMANS and P. J. BRUSSAARD \\ Fysisch Laboratorium der Rijksuniversiteit, Utrecht, Netherlands \\ and \\ B. H. WILDENTHAL ${ }^{+}$ \\ Oak Ridge National Laboratory, Oak Ridge, Tennessee
}

Received 25 May 1967

\begin{abstract}
The addition of a $T$-dependent but $J$-independent term to the surface delta interaction greatly improves the agreement between the values of the two-body matrix elements calculated from this interaction and the corresponding values obtained both from empirical fits to level energies and from a realistic (Hamada-Johnston) interaction. This is shown for $1 \mathrm{~d}-2 \mathrm{~s}$ and $1 \mathrm{f}-2 \mathrm{p}$ configurations. The use of this modified surface delta interaction as a shell-model residual interaction produces good agreement between the calculated and experimental energies of manynucleon configurations. In particular, total binding energies and the energy spacings between levels of different isospin are fitted much better than is possible without the use of the extra $T$-dependent term.
\end{abstract}

\section{Introduction}

Shell-model calculations have been performed recently with the surface delta interaction ${ }^{1}$ ) (SDI) as an effective two-body interaction in the ds shell ${ }^{2,3}$ ) and for heavier nuclei ${ }^{4}$ ). It was found that the energy spacings between low-lying levels of a given value of the mass $A$ and of isospin $T$ could be quite well reproduced with this interaction. In this paper it will be shown that the addition of a $T$-dependent but $J$-independent term to the SDI greatly improves the agreement with (i) the experimentally observed binding energies and excitation energies, including those of different isospin, (ii) the two-body matrix elements that are obtained empirically from a leastsquares fit to level energies, and (iii) the two-body matrix elements that are calculated from a realistic (Hamada-Johnston) potential.

The SDI is defined ${ }^{4}$ ) as

$$
V_{\mathrm{SDI}}(i j)=-4 \pi A_{T} \delta\left(\Omega_{i j}\right) \delta\left(r_{i}-R\right) \delta\left(r_{j}-R\right),
$$

where $\Omega_{i j}$ is the angular coordinate between the interacting particles $i$ and $j$, and $R$ the nuclear radius. The two strengths, $A_{1}$ for a $T=1$ coupled pair and $A_{0}$ for $T=0$, are the only parameters that enter the expression of the two-body matrix elements.

† U.S.A.E.C. Postdoctoral Fellow under appointment from Oak Ridge Associated Universities. 
An anti-symmetrized two-body matrix element of the SDI can be written in $j-j$ coupling as

$$
\begin{aligned}
\left\langle j_{\mathrm{a}} j_{\mathrm{b}}\left|V_{\mathrm{SDI}}\right| j_{\mathrm{c}} j_{\mathrm{d}}\right\rangle_{J}, T & \frac{A_{T}}{2(2 J+1)}\left\{\frac{\left(2 j_{\mathrm{a}}+1\right)\left(2 j_{\mathrm{b}}+1\right)\left(2 j_{\mathrm{c}}+1\right)\left(2 j_{\mathrm{d}}+1\right)}{\left(1+\delta_{\mathrm{ab}}\right)\left(1+\delta_{\mathrm{cd}}\right)}\right\}^{\frac{1}{2}} \\
\times & {\left[(-1)^{l_{\mathrm{a}}+l_{\mathrm{c}}+j_{\mathrm{b}}+j_{\mathrm{d}}}\left\langle j_{\mathrm{a}} \frac{1}{2} j_{\mathrm{b}}-\frac{1}{2} \mid J 0\right\rangle\left\langle j_{\mathrm{c}} \frac{1}{2} j_{\mathrm{d}}-\frac{1}{2} \mid J 0\right\rangle\left\{1-(-1)^{l_{\mathrm{c}}+l_{\mathrm{d}}+J+T}\right\}\right.} \\
& \left.-\left\langle j_{\mathrm{a}} \frac{1}{2} j_{\mathrm{b}} \frac{1}{2} \mid J 1\right\rangle\left\langle j_{\mathrm{c}} \frac{1}{2} j_{\mathrm{d}} \frac{1}{2} \mid J 1\right\rangle\left\{1+(-1)^{T}\right\}\right],
\end{aligned}
$$

where $J$ and $T$ are the spin and isospin of the two-particle system, $j_{k}$ and $l_{k}$ the spin and the orbital angular momentum of a particle in orbit $k$ and $\left\langle j_{1} m_{1} j_{2} m_{2} \mid J M\right\rangle$ a Clebsch-Gordan coefficient. The small number of adjustable parameters makes the SDI a very convenient residual interaction for use in shell-model calculations. However, the position of $T>T_{z}$ states with respect to the position of $T=T_{z}$ states cannot be well reproduced by the SDI given in eq. (1), as will be shown below.

\section{Modification of the SDI}

Consider two groups of energy levels of one nucleus, one group with $T=T_{1}$ and the other with $T=T_{2}$. Let $S_{12}(\exp )$ be the experimental value of the energy spacing between the lowest state with $T=T_{1}$ and the lowest state with $T=T_{2}$, and let $S_{12}$ (calc) be the theoretical value of this spacing as reproduced with the SDI. Let us denote the difference between the experimental and calculated values by $\Delta_{T_{1} T_{2}} \equiv$ $S_{12}(\exp )-S_{12}$ (calc).

The values of $S_{12}(\mathrm{exp})$ are taken from the experimentally determined excitation energies of the lowest analogue state. When these energies were not known experimentally, they were calculated with an expression for Coulomb energies given by deShalit and Talmi ${ }^{7}$ ). This expression has been used successfully in previous calculations ${ }^{8}$ ). The values $S_{12}$ (calc) are derived from shell-model calculations in which the strengths $A_{1}$ and $A_{0}$ (see eq. (1)) were determined such that the calculated energies of the many-nucleon configurations yielded a least-squares fit to experimentally observed energies. Only the excitation energies of levels of a given $T$ with respect to the lowest state of the same $T$ were fitted in many nuclei simultaneously and an average of about three low-lying levels in each nucleus was used. No attempts were made to fit the binding energies of the ground states of the various nuclei.

It is found that the values of $\Delta_{T_{1} T_{2}}$ are rather independent of mass number $A$. This is illustrated for the nuclei in the mass region $30 \leqq A \leqq 38$ in fig. 1 , where $\Delta_{T_{1} T_{2}}$ has been plotted against $A$. In table 1 mean values of $\Delta_{T_{1} T_{2}}$ are listed. These mean values were obtained by averaging $\Delta_{T_{1} T_{2}}$ over the nuclei in the three non-overlapping intervals $18 \leqq A \leqq 22,23 \leqq A \leqq 28$ and $30 \leqq A \leqq 38$.

In the first two intervals the $1 \mathrm{~d}_{\frac{5}{2}}$ and $2 \mathrm{~s}_{\frac{1}{2}}$ subshells were taken into account and in the third interval the $2 \mathrm{~s}_{\frac{1}{2}}$ and $1 \mathrm{~d}_{\frac{3}{2}}$ subshells. The rather different values of $\bar{\Delta}_{T_{1} T_{2}}$ in 
the second interval are presumably caused by the neglect of the $1 \mathrm{~d}_{\frac{3}{2}}$ subshell in the model calculations.

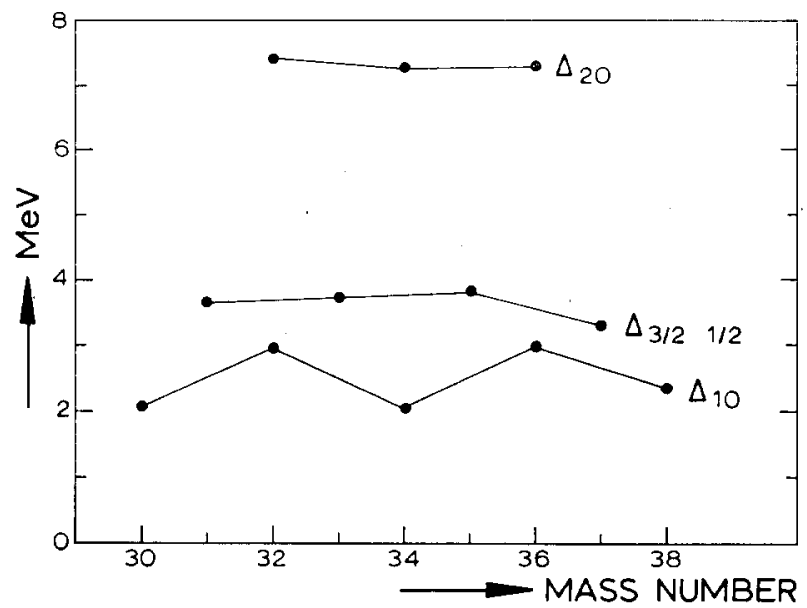

Fig. 1. Mass dependence of the deviations $A_{T_{1} T_{2}}$ for $s_{\frac{1}{2}} d_{\frac{3}{2}}$ shell nuclei. The definition of $\Delta_{T_{1} T_{2}}$ is given in sect. 2 .

TABLE 1

Values (in MeV) of $\bar{A}_{T_{1} T_{2}}$ and $D_{T_{1} T_{2}}$ for ds shell nuclei

\begin{tabular}{lccccccccc}
\hline Mass region & Configuration & $A_{1}$ & $A_{0}$ & $\bar{J}_{10}$ & $D_{10}$ & $\bar{\Delta}_{20}$ & $D_{20}$ & $\bar{\Delta}_{\frac{3}{2} \frac{1}{2}}$ & $D_{\frac{3}{2} \frac{1}{2}}$ \\
\hline $18 \leqq A \leqq 22$ & $\mathrm{~d}_{\frac{3}{2}} \mathrm{~s}_{\frac{1}{2}}$ & 1.0 & 1.3 & 2.6 & 2.4 & 7.2 & 7.2 & 3.3 & 3.6 \\
$23 \leqq A \leqq 28$ & $\mathrm{~d}_{\frac{5}{2}} \mathrm{~s}_{\frac{1}{2}}$ & 1.1 & 0.3 & 4.3 & 3.9 & 11.2 & 11.7 & 5.9 & 5.9 \\
$30 \leqq A \leqq 38$ & $\mathrm{~s}_{\frac{1}{2}} \mathrm{~d}_{\frac{3}{2}}$ & 1.2 & 0.4 & 2.4 & 2.4 & 7.3 & 7.2 & 3.6 & 3.6
\end{tabular}

The values of $A_{1}$ and $A_{0}$ represent the strengths of the SDI as obtained by fitting excitation energies in the various mass regions. The meaning of the symbols $\bar{\Delta}_{T_{1} T_{2}}$ and $D_{T_{1} T_{2}}$ is explained in the text.

Analysis shows that the deviations $\Delta_{T_{1} T_{2}}$ can be compensated for by adding a $T$-dependent term to the SDI defined in eq. (1). Thus we get

$$
V_{i j}=-4 \pi A_{T} \delta\left(\Omega_{i j}\right) \delta\left(r_{i}-R\right) \delta\left(r_{j}-R\right)+B_{T}
$$

In terms of two-body matrix elements this modification amounts to adding a term $B_{T}$ to the diagonal matrix elements only. The two parameters $B_{1}$ and $B_{0}$ of the modified surface delta interaction (MSDI), eq. (3), do not depend on $J$ but only affect the energy spacing between the groups of $T=T_{1}$ and $T=T_{2}$ states. There is a simple relation between $\Delta_{T_{1} T_{2}}$ and the parameters $B_{T}$. In order to derive this relation, let us consider a diagonal matrix element of the interaction between $k$ particles occupying the shells $\rho_{1} \ldots \rho_{k}$ outside a core. This matrix element can be expressed in terms of 
two-body matrix elements as

$$
\begin{aligned}
\left\langle\rho_{1} \ldots \rho_{k}|H| \rho_{1} \ldots \rho_{k}\right\rangle_{J T}=\sum_{n} c_{n}^{(1)} x_{n}\left(T^{\prime}=1\right)+\sum_{m} c_{m}^{(0)} x_{m}\left(T^{\prime}=0\right) & \\
& + \text { single-particle terms. }
\end{aligned}
$$

Here $x_{n}\left(T^{\prime}=1\right)$ and $x_{m}\left(T^{\prime}=0\right)$ denote the two-particle matrix elements for pairs coupled to $T^{\prime}=1$ and $T^{\prime}=0$, respectively. The coefficients $c_{n}^{(1)}$ and $c_{m}^{(0)}$ are derived with standard Racah techniques ${ }^{8}$ ). The sums of these coefficients, $\sum_{n} c_{n}^{(1)}$ and $\sum_{m} c_{m}^{(0)}$, give the number of pairs $T^{\prime}=1$ and $T^{\prime}=0$, respectively, that are present in the original $k$-particle configuration $\left|\rho_{1} \ldots \rho_{k}\right\rangle_{J T}$. The two-body operator $V_{i j}=\frac{1}{2} T^{2}$ with $T^{\prime}=t_{i}+t_{j}$ has eigenvalues 1 and 0 for pairs $T^{\prime}=1$ and $T^{\prime}=0$, respectively. Therefore

$$
\begin{aligned}
\sum_{n} c_{n}^{(1)} & =\left\langle\rho_{1} \ldots \rho_{k}\left|\sum_{i<j}^{k} V_{i j}\right| \rho_{1} \ldots \rho_{k}\right\rangle_{J T} \\
& =\frac{1}{2}\left\langle\rho_{1} \ldots \rho_{k}\left|T^{2}-\sum_{i} t_{i}^{2}+\sum_{i<j}\left(t_{i}^{2}+t_{j}^{2}\right)\right| \rho_{1} \ldots \rho_{k}\right\rangle_{J T} \\
& =\frac{3}{8} k(k-2)+\frac{1}{2} T(T+1)
\end{aligned}
$$

where $\boldsymbol{T}=\sum_{i} \boldsymbol{t}_{i}$.

From the relation $\sum_{n} c_{n}^{(1)}+\sum_{m} c_{m}^{(0)}=\left(\begin{array}{l}k \\ 2\end{array}\right)=\frac{1}{2} k(k-1)$, it follows immediately that

$$
\sum_{m} c_{m}^{(0)}=\frac{1}{8} k(k+2)-\frac{1}{2} T(T+1) \text {. }
$$

From eqs. (4)-(6), we see that the addition of the two terms $B_{1}$ and $B_{0}$ in eq. (3) changes the value of the diagonal $k$-particle matrix elements of isospin $T$ by an amount of

$$
\left[\frac{3}{8} k(k-2)+\frac{1}{2} T(T+1)\right] B_{1}+\left[\frac{1}{8} k(k+2)-\frac{1}{2} T(T+1)\right] B_{0} .
$$

This variation is independent of the spin $J$ of the $k$-particle state. The off-diagonal $k$-particle matrix elements are not affected by the terms $B_{1}$ and $B_{0}$, so that also in case of configuration mixing the $B$-terms will shift the calculated level energies independently of $J$, as given in eq. (7). This implies that, for a fixed value of $k$, the positions of the $T=T_{1}$ levels are shifted with respect to the $T=T_{2}$ levels by an amount of

$$
D_{T_{1} T_{2}}=\frac{1}{2}\left[T_{1}\left(T_{1}+1\right)-T_{2}\left(T_{2}+1\right)\right]\left(B_{1}-B_{0}\right),
$$

as follows directly from eq. (7).

Eq. (8) reproduces the deviation $\Delta_{T_{1} T_{2}}$ very well with a single value of $B_{1}-B_{0}$, as is demonstrated for the mean values $\bar{\Delta}_{T_{1} T_{2}}$ in table 1 for three combinations of $T_{1}$ and $T_{2}$ [note that $B_{1}-B_{0}=D_{10}$ according to eq. (8)]. Thus the MSDI not only renders the spectra of the $T=T_{z}$ and $T>T_{z}$ levels (as did the unmodified SDI) but also the correct spacings of these groups of levels with respect to each other.

We still have the value of $B_{1}$ or $B_{0}$ (their difference has been fixed above) at our 
disposal to fit the binding energies of nuclei of different mass number. Addition of an amount $b$ to both $B_{1}$ and $B_{0}$ will result in a shift of all levels of a nucleus (with $k$ particles outside the core) given by $\frac{1}{2} k(k-1) b$. With this extra degree of freedom it proves to be possible to fit the binding energies of the lowest state of each mass number.

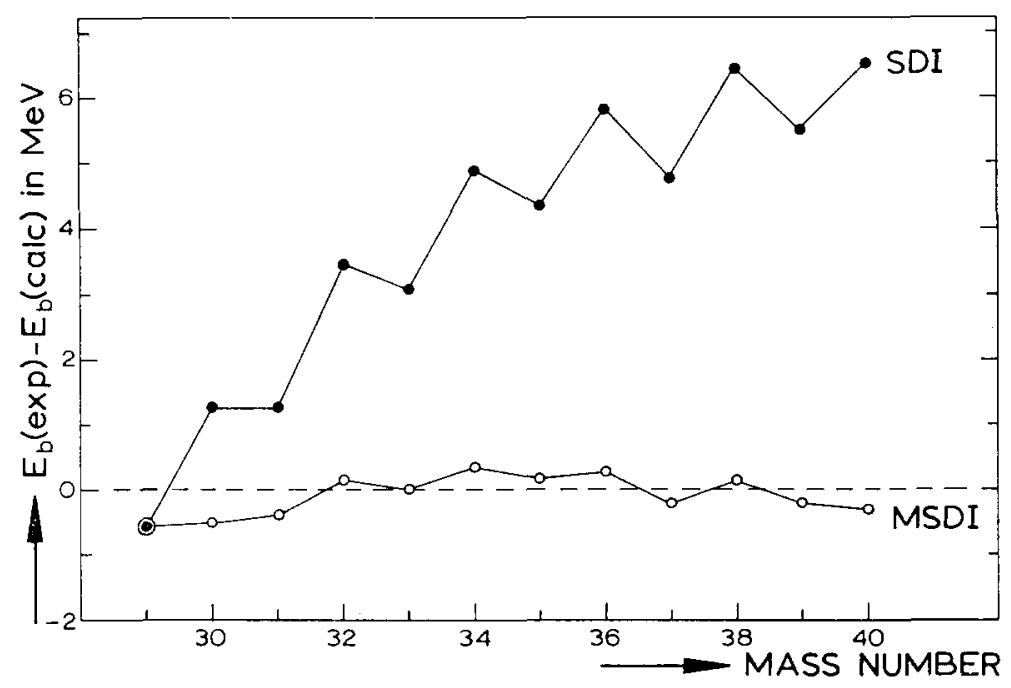

Fig. 2. The differences between the experimental and calculated binding energies $E_{\mathbf{b}}$ (with $E_{\mathbf{b}}>0$ ) are shown for $B_{1}=0.7 \mathrm{MeV}$ and $B_{0}=-1.8 \mathrm{MeV}$ (plot marked MSDI) and for $B_{1}=B_{0}=0$ (plot marked SDI).

The best fit for binding energies as well as level spacings in the $29 \leqq A \leqq 40$ mass region is obtained for $A_{1}=1.2 \mathrm{MeV}, A_{0}=0.4 \mathrm{MeV}, B_{1}=0.7 \mathrm{MeV}$ and $B_{0}=-1.8$ $\mathrm{MeV}$. In the least-squares fitting procedure 23 ground states and 35 excited states have been used. The agreement for the ground-state binding energies is illustrated by the plot marked MSDI in fig. 2 for the even-mass $(T=0)$ and the odd-mass $\left(T=\frac{1}{2}\right)$ nuclei. The agreement for the excitation energies is of the same quality as for the binding energies. The deviations between the experimental and calculated binding energies for $B_{1}=B_{0}=0$ are shown in the same figure by the plot marked SDI. In the latter case all other parameters, i.e. the strengths $A_{1}$ and $A_{0}$ as well as the single-particle energies, are kept equal to those of the MSDI fit.

It is clear that the deviations of the calculated binding energies according to the SDI, which are seen to be non-linear in the number of active particles, cannot be well absorbed into the single-particle energies. In the first place a change in the singleparticle energies would result in a shift of the binding energies linear in the number of active particles and secondly such a change could not correct the systematic differences obtained between odd-mass and even-mass nuclei. 


\section{Comparison of the MSDI with empirical two-body matrix elements}

Numerical values of two-body matrix elements can be derived in a shell-model calculation from experimental nuclear level spectra. The two-body matrix elements are considered as free parameters to be determined from a least-squares fitting procedure to the energy levels.

It is interesting to compare such empirical matrix elements with those calculated with the MSDI.

\subsection{MATRIX ELEMENTS OF $s_{\frac{1}{2}} d_{\frac{3}{2}}$ AND $d_{\frac{3}{2}} f_{\frac{7}{2}}$ CONFIGURATIONS}

Empirical two-body matrix elements obtained in the $2 \mathrm{~s}_{\frac{1}{2}} 1 \mathrm{~d}_{\frac{3}{2}}$ subshells ${ }^{2}$ ) and in the $1 \mathrm{~d}_{\frac{3}{2}} 1 \mathrm{f}_{\frac{7}{2}}$ subshells ${ }^{5}$ ) are compared with those of the MSDI in fig. 3 . The values of the MSDI two-body matrix elements are calculated with the values of $A_{1}, A_{0}, B_{1}$ and $B_{0}$ that were determined in a least-squares fit to experimental energies of $s_{\frac{1}{2}}^{n} \mathrm{~d}_{\frac{3}{2}}^{m}$ configurations (see sect. 2).

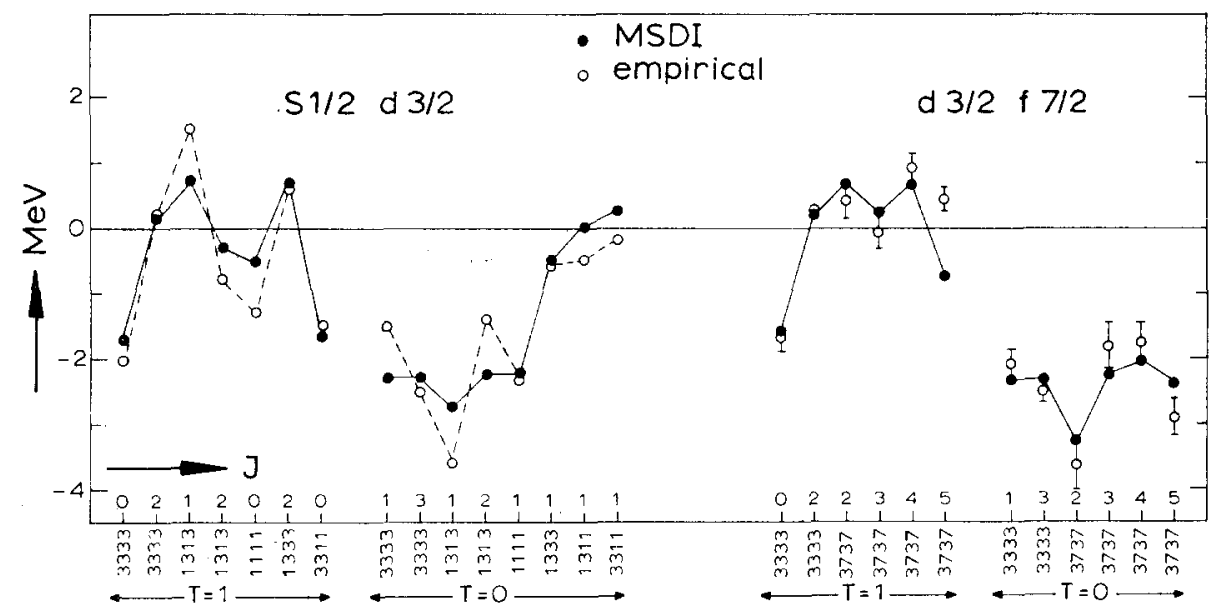

Fig. 3. Values of two-body matrix elements calculated from the MSDI with $A_{1}=1.2 \mathrm{MeV}, A_{0}=0.4$ $\mathrm{MeV}, B_{1}=0.7 \mathrm{MeV}$ and $B_{0}=-1.8 \mathrm{MeV}$ are compared with matrix elements obtained empirically. The $n l_{j}$ values are specified by 1 for $2 \mathrm{~s}_{\frac{1}{2}}, 3$ for $1 \mathrm{~d}_{\frac{3}{2}}$ and 7 for $1 \mathrm{f}_{\frac{7}{2}}$.

Some agreement between MSDI and empirical two-body matrix elements for the $s_{\frac{1}{2}} d_{\frac{3}{2}}$ configurations should be expected, since both reproduce the same experimental spectra. It is remarkable to see, however, that also for $\mathrm{d}_{\frac{3}{2}} \mathrm{f}_{\frac{7}{2}}$ configurations the two sets of matrix elements agree very well, especially since the values of $A_{1}, A_{0}, B_{1}$ and $B_{0}$ are taken exactly the same as those used for the $\mathrm{s}_{\frac{1}{2}} \mathrm{~d}_{\frac{3}{2}}$ matrix elements.

\subsection{MATRIX ELEMENTS OF THE $f_{\frac{3}{2}} \mathrm{p}_{\frac{3}{2}}$ CONFIGURATION}

Values of the MSDI parameters $A_{1}$ and $B_{1}$ can be determined by a least-squares fitting procedure to levels of the ${ }^{41} \mathrm{Ca}$ to ${ }^{48} \mathrm{Ca}$ isotopes with $\mathrm{f}_{\frac{7}{2}}^{n} \mathrm{p}_{\frac{3}{2}}^{m}$ configurations. The 
best fit to eight ground states and 20 low-lying excited states is obtained with $A_{1}=0.6$ $\mathrm{MeV}, B_{1}=0.2 \mathrm{MeV}$. The average deviation between experimentally determined and calculated energies of ground states and levels was found to be $0.2 \mathrm{MeV}$.

Values of empirical two-body matrix elements for $\mathrm{f}_{\frac{7}{2}} \mathrm{p}_{\frac{3}{2}}$ configurations have been obtained, e.g. by Engeland and Osnes ${ }^{9}$ ) and by Federman and Talmi ${ }^{10}$ ). In fig. 4 these empirical matrix elements are compared with those calculated from the MSDI fit. There is again a good correspondence between the MSDI and empirical values of almost all matrix elements.

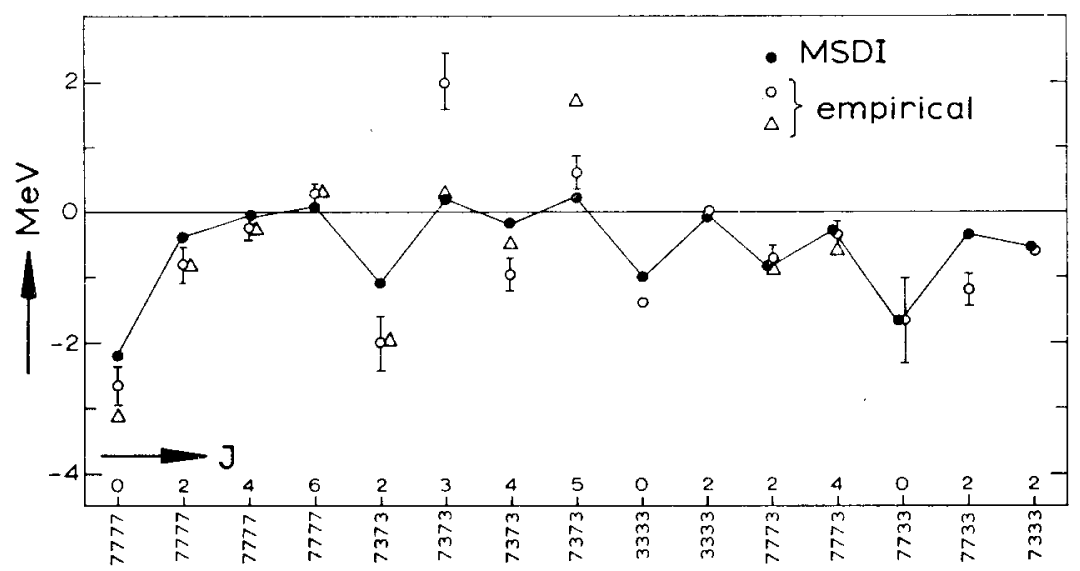

Fig. 4. Values of the $T=1$ two-body matrix elements calculated from MSDI with $A_{1}=0.6 \mathrm{MeV}$ and $B_{1}=0.2 \mathrm{MeV}$ are compared with matrix elements obtained empirically. The values indicated by the triangles are from ref. ${ }^{9}$ ), the open circles are from ref. ${ }^{10}$ ). The $n l j$ values are specified by 7 for $1 \mathrm{f}_{\frac{7}{2}}$ and 3 for $1 \mathrm{p}_{\frac{3}{2}}$.

Unfortunately no empirical $T=0$, two-body matrix elements are available for comparison, so that we have not attempted to determine the parameters $A_{0}$ and $B_{0}$ for $f_{\frac{3}{2}} p_{\frac{3}{2}}$ configurations.

\section{Comparison of the MSDI with matrix elements from a realistic potential}

Recently, progress has been made in deducing two-body matrix elements from a realistic two-body interaction with a hard core such as the Hamada-Johnston potential. Such results for $T=1$ two-body matrix elements of $\mathrm{p}_{\frac{3}{2}} \mathrm{p}_{\frac{1}{2}} \mathrm{f}_{\frac{5}{2}}$ configurations ${ }^{6}$ ) are compared in fig. 5 with the MSDI matrix elements, calculated for the same values of $A_{1}$ and $B_{1}$ as were given in subsect. 3.2.

It should be noted that the quoted numerical values of the two-body matrix elements of the Hamada-Johnston potential are the results of a lengthy calculation in which very appreciable corrections due to core excitations ${ }^{6}$ ) had to be taken into account. The MSDI, by showing agreement with these corrected matrix elements, appears to 
be an effective interaction that reproduces these matrix elements with surprisingly few parameters. Comparison of the MSDI matrix elements with $T=0$ two-body matrix elements of the Hamada-Johnston potential for $\mathrm{p}_{\frac{3}{2}} \mathrm{p}_{\frac{1}{2}} \mathrm{f}_{\frac{3}{2}}$ configurations has been possible with some preliminary values ${ }^{11}$ ) of the matrix elements of the realistic interaction. The reproduction by the MSDI was fairly good (with $A_{0}=0.4 \mathrm{MeV}, B_{0}=$ $-0.5 \mathrm{MeV}$ ), but not as good as for the $T=1$ case.

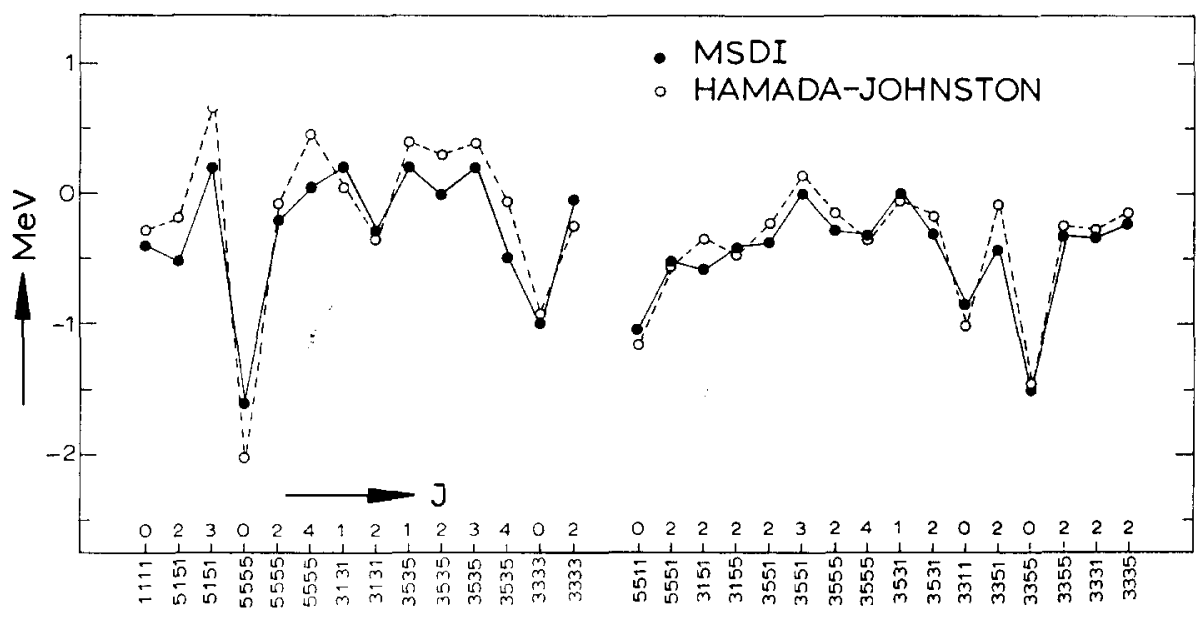

Fig. 5. Values of the $T=1$ two-body matrix elements in the fp shell calculated from a MSDI fit to energy levels of the $\mathrm{Ca}$ isotopes $\left(A_{1}=0.6 \mathrm{MeV}, B_{1}=0.2 \mathrm{MeV}\right)$ are compared with matrix elements obtained $^{6}$ ) from a realistic (Hamada-Johnston) potential. The $n l j$ values are given by 3 for $2 \mathrm{p}_{\frac{3}{2}}$, 1 for $2 \mathrm{p}_{\frac{1}{2}}$ and 5 for $1 \mathrm{f}_{\frac{5}{2}}$.

\section{Discussion}

The MSDI has been applied in the previous sections to ds and fp shell nuclei. The four parameters $A_{1}, B_{1}, A_{0}$ and $B_{0}$ were deduced from a direct fit to nuclear levels. The resulting two-body matrix elements were compared with matrix elements that were obtained (i) empirically and (ii) from a realistic potential. For other mass regions we have not attempted to make a direct fit to nuclear level schemes.

It was found, however, that the empirical two-body matrix elements of the $1 p$ shell ${ }^{12}$ ) (for $T=0$ and $T=1$ ) and of the $\lg _{\frac{9}{2}} 2 \mathrm{p}_{\frac{1}{2}}$ subshells ${ }^{13}$ ) (for $T=1$ ) could also be fairly well described with the MSDI parameters given by (values in $\mathrm{MeV}$ ) $A_{1}=3.0, B_{1}=2.0, A_{0}=2.6, B_{0}=-1.0$, and $A_{1}=0.5, B_{1}=0.6$, respectively.

For all cases considered the ratio of the strengths $A_{1} / A_{0}$ was found to be $>0.8$. If one uses one of the customary nuclear-force mixtures, such as, e.g., Rosenfeld, Soper, Sliv or Gillet, one finds for the ratio of the strengths of the $T=1$ over the $T=0$ interaction a value of about 0.5 . However, since the values of all SDI diagonal matrix elements are $\leqq 0$, the contribution of the negative term $B_{0}$ will enlarge the magnitude of the diagonal $T=0$ matrix elements. The contribution of the positive 
term $B_{1}$ diminishes the magnitude of almost all diagonal $T=1$ matrix elements.

It is worthwhile to remark that the addition of the term $B_{1}$ to the particle-particle interaction will shift the diagonal particle-hole matrix elements $\left\langle\rho_{1} \rho_{2}^{-1}\left|V_{12}\right| \rho_{1} \rho_{2}^{-1}\right\rangle_{J T}$ by the amount $-\frac{1}{2}\left(B_{1}+B_{0}\right)$ for $T=1$ and by $-\frac{1}{2}\left(3 B_{1}-B_{0}\right)$ for $T=0$. Isospindependence of particle-hole interactions has been discussed in refs. ${ }^{14,15}$ ).

The authors are very much indebted to Dr. E. C. Halbert and Dr. J. B. McGrory for their permission to make use of their shell-model computer codes and for many helpful conversations.

\section{References}

1) R. Arvieu and S. A. Moszkowski, Phys. Rev. 145 (1966) 830

2) P. W. M. Glaudemans, B. H. Wildenthal and J. B. McGrory, Phys. Lett. 21 (1966) 427

3) B. H. Wildenthal, P. W. M. Glaudemans, E. C. Halbert and J. B. McGrory, Bull. Am. Phys. Soc. 12 (1967) 48

4) A. Plastino, R. Arvieu and S. A. Moszkowski, Phys. Rev. 145 (1966) 837

5) F. C. Erné, Nuclear Physics 84 (1966) 91

6) R. D. Lawson, M. H. Macfarlane and T. T. S. Kuo, Phys. Lett. 22 (1966) 168

7) A. de-Shalit and I. Talmi, Nuclear shell theory (Academic Press, New York, 1963) eq. (30.2)

8) P. W. M. Glaudemans, G. Wiechers and P. J. Brussaard, Nuclear Physics 56 (1964) 529

9) T. Engeland and E. Osnes, Phys. Lett. 20 (1966) 424

10) P. Federman and I. Talmi, Phys. Lett. 22 (1966) 466

11) T. T. S. Kuo, private communication

12) S. Cohen and D. Kurath, Nuclear Physics 73 (1965) 1

13) S. Cohen, R. D. Lawson, M. H. Macfarlane and M. Soga, Phys. Lett. 10 (1964) 195

14) R. K. Bansal and J. B. French, Phys. Lett. 11 (1964) 145

15) L. Zamick, Phys. Lett. 19 (1965) 580 